\title{
THE APPLICATION OF PASSIVE THERMOGRAPHY AND MEASUREMENT OF BURN SURFACE AREA FOR THE ASSESSMENT OF THE BURN WOUND HEALING PROCESS.
}

\author{
M. Dziewonski ${ }^{1}$
}

${ }^{1}$ Department of Strength of Materials and Computational Mechanics, Silesian University of Technology, Gliwice, Poland (miroslaw.dziewonski@polsl.pl)

\begin{abstract}
Defining of the progress in burn treatment is not an easy task, as burn wounds look similar or even identical at all stages of dressings and therapeutic treatments. In most cases, the assessment of the wound healing is based on the photographs taken at particular stages of treatment. In addition, the measurement of surface area of the wound indicated by the doctor is used to make the assessment more objective.

Due to the fact that some changes are not visible on the surface of the wound, it is essential to provide thermograms, which show the variation of temperatures on the wound surface. The quantity assessment, connected for example with the surface area measurement, is very difficult. Thermographic pictures on average do not have tight edges allowing for an identification of borders between the areas with pathological changes and the undamaged tissue. Therefore, in practice the damaged areas are identified on the basis of the temperature variation between the area of the injury and the undamaged tissue with a reference to a particular temperature.

The article will discuss the algorithms enabling the measurement of the surface area in thermograms and their application allowing for an easier and more objective assessment of the progress of the injury treatment as well as making initial diagnosis of abnormalities in the process.
\end{abstract}

Keywords: Thermography, Diagnosis, Image analysis, Chronic wound, Burns

\section{INTRODUCTION}

The process of burn and chronic wound treatment is arduous, costly and prolonged. The possibility of qualitative and quantitative monitoring of the healing process is very important for the optimum management. A properly made diagnosis and related medical procedures can effectively reduce the time and costs of patients' treatment.

The assessment of the wound healing is based, inter alia, on the photographs taken at particular stages of treatment. In addition, the measurement of surface area of the wound indicated by the doctor is used to make the assessment more objective. 
Due to the fact that some changes are not visible on the surface of the wound, it is essential to provide thermograms (fig. 1). The temperature of tissue is closely associated with its blood supply, the level of blood supply corresponds to the condition of tissue and reflects its lesions.
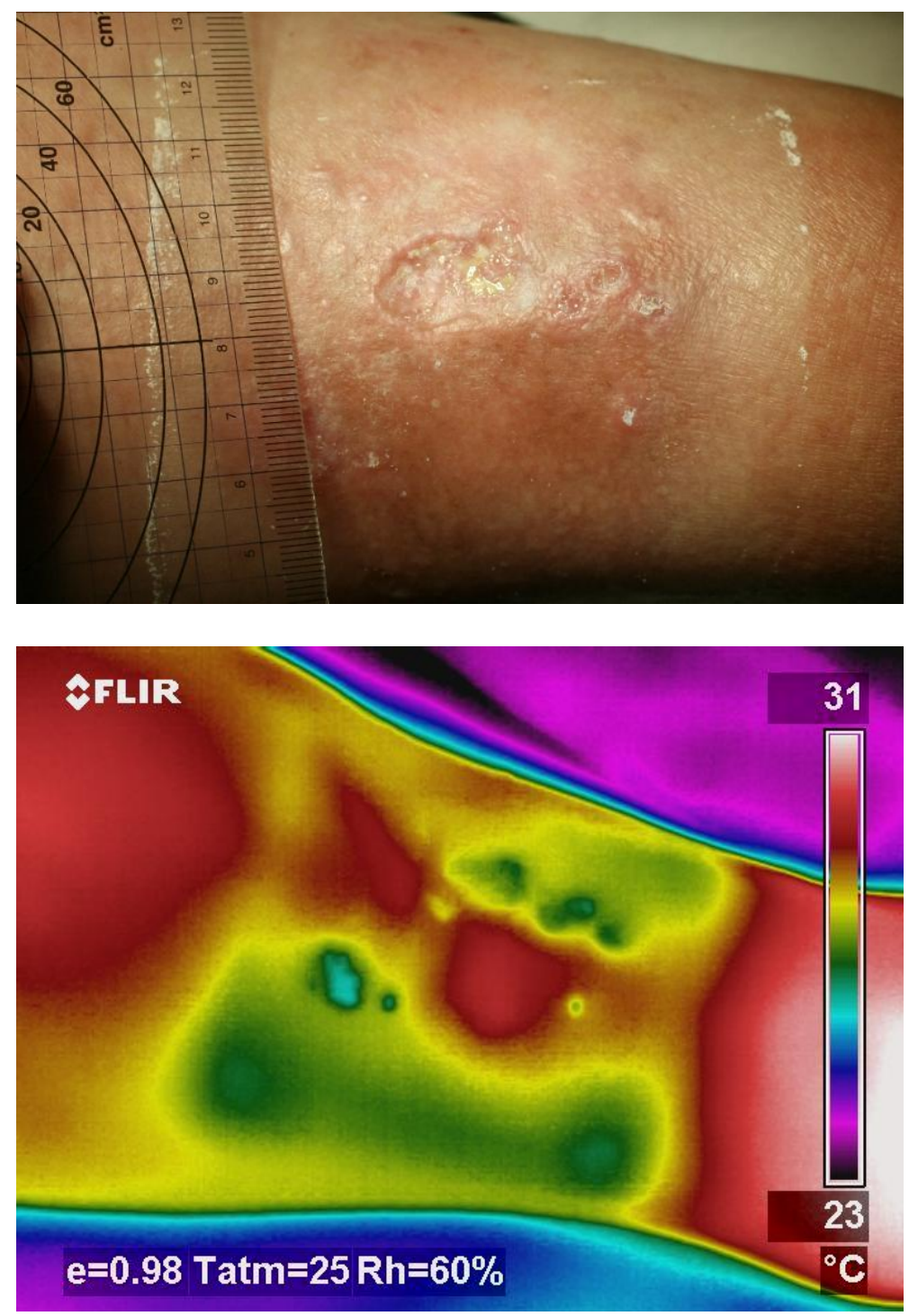

Figure 1. Photo of the wound and its thermal image.

As a result of technology development and the price reduction of thermal imaging equipment (cameras and scanners), thermography becomes more and more popular diagnostic tool in many medical centers treating burns $[4,5,8]$. It is used primarily to specify the grade of burn and the areas of necrosis. The equipment records flat thermal images (thermograms), and the data obtained are mainly used for qualitative assessment [6, 7].

In addition, there is also a diagnostic method using the Doppler effect [2], but it does not allow for any accurate injury diagnostics and the assessment of the lesion dynamics that occurs within the wound (Doppler effect signals cannot differentiate infectious lesions from neoangiogenesis). 
The quantity assessment, connected with the surface area measurement, in case of classical thermograms is very difficult and for larger wounds even impossible (fig. 2). Thermographic pictures on average do not have tight edges allowing for an identification of borders between the areas with pathological changes and the undamaged tissue.
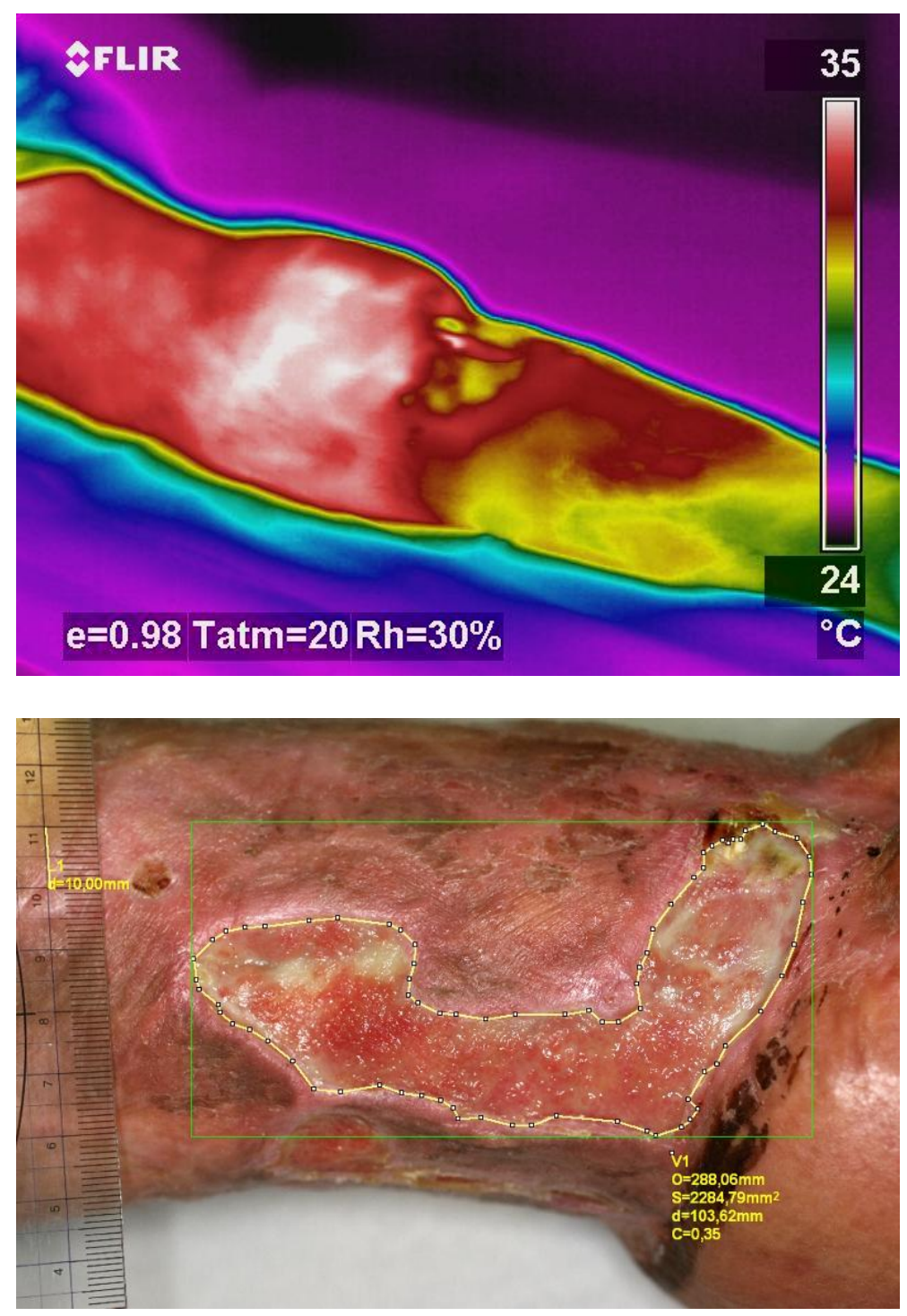

Figure 2. Comparison of the wound surface in the thermogram and photo.

Therefore, apart from the specific algorithms of surface measurement for photographs and thermograms, a prototype diagnosing system, enabling thermographic measurements for larger wounds, will be presented in the further part of the article.

\section{MEASUREMENT OF THE WOUND SURFACE}

While measuring the surface of the areas marked in the digital photo, commonly known algorithms are used. The same algorithms are also applied for example in geodesy.

The first stage of wound measurement and calculation process is point digitalization which involves marking the given points with a cursor by an operator. When matched togeth- 
er, the points make a contour, which is the boundary of the burn area (fig. 3).

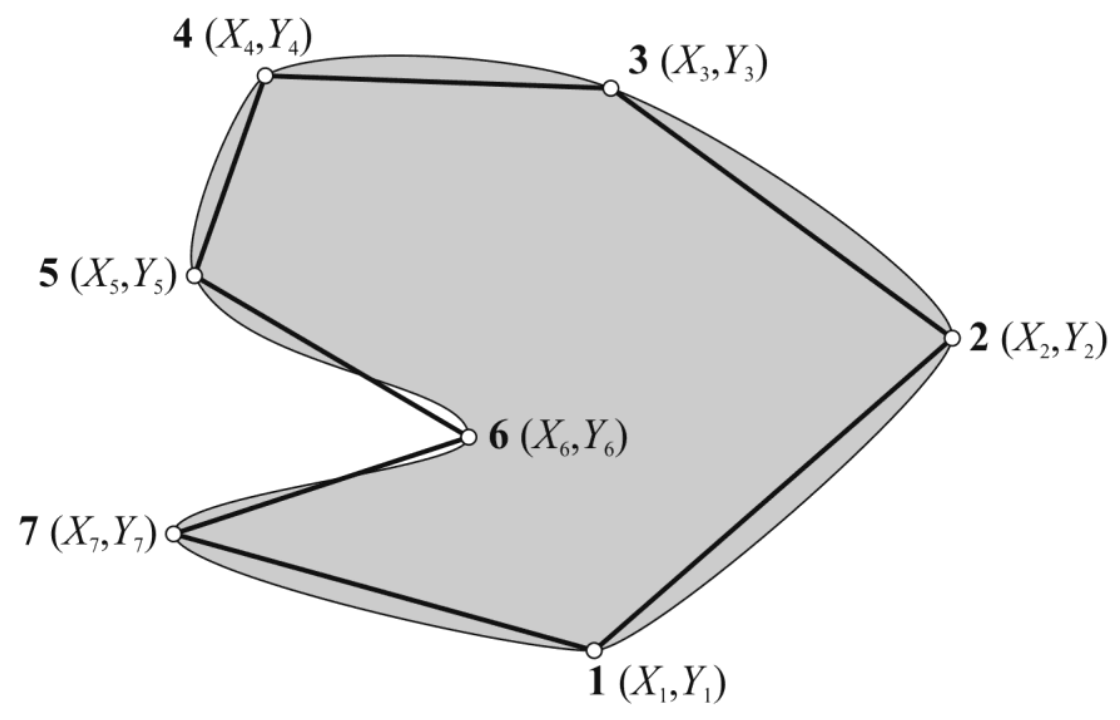

Figure 3. Point digitizing.

While creating applications, the focus was on the linear contours, since in practice it turned out that applying other contours e.g. using Bezier curve or NURBS is inconvenient for system operators.

The digitized coordinates of the points identified are recorded and on this basis the surface area $P$ is calculated using the following formulas:

$$
2 P=\sum_{i=1}^{n} X_{i}\left(Y_{i+1}-Y_{i-1}\right)
$$

where $n$ is the number of vertices, $Y_{n+1}=Y_{1}, Y_{0}=Y_{n}$ and

$$
2 P=\sum_{i=1}^{n} Y_{i}\left(X_{i+1}-X_{i-1}\right)
$$

where $X_{n+1}=X_{1}$ and $X_{0}=X_{n}$.

The above formulas should be used collectively, as they make up a mutual calculation control.

Received values of the marked surface area should be calculated basing on an appropriate scale $S$. It can be determined in two ways:

- by setting the frame size based on the "optics geometry" of a thermographic camera and the distance from the object;

- by indicating a known segment and comparing it to the real dimension. The best way to do that is by taking an image along with the rule. 


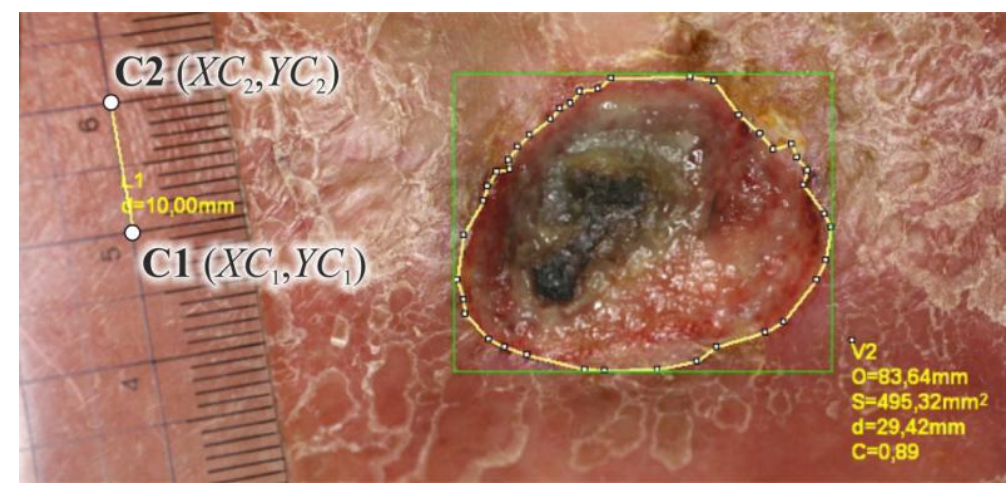

Figure 4. Photo of the wound together with the rule.

Using the second method, it is necessary to mark two control points on the image, indicating the ends of the model segment (fig. 4). Based on their coordinates $\left(X C_{1}, Y C_{1}, X C_{2}\right.$, $Y C_{2}$ ), scale $S$ is set from the following dependence:

$$
S=\frac{L}{\sqrt{\left(X C_{2}-X C_{1}\right)^{2}+\left(Y C_{2}-Y C_{1}\right)^{2}}}
$$

where $L$ is the known length of the model segment.

Multiplying the area $P$ given by the formula (1) or (2) by the square of the calculated scale $S(3)$ we receive the actual surface area of the marked wound area $P_{W}$ :

$$
P_{W}=P \cdot S^{2}
$$

Using point digitalization in analyzing thermograms is difficult because, as it has already been mentioned, the transition between the areas of various temperatures is vague, without clear boundaries. Therefore, hand-held attempts of indicating the areas are strenuous and prone to serious mistakes.

Having consulted a group of doctors, it was assumed that in case of thermograms, the parameter contributing to the assessment of wound healing progress can be the measurement of the surface area of similar temperatures in the subareas suspected of resulting in pathological changes [1].

A thermogram is a dispersion of radiation intensity measured by the detector's particular cells (e.g. microbolometers) and registered in two dimensional arrays having the measurements consistent with the detector's resolution (e.g. 640x480). Each pixel (an element of the array) can be presented either in an arithmetic form, for example showing the existing temperatures of an object, or in a binary form. In the latter case, in order to read the registered temperatures it is essential to know the temperature range set in the camera during the measurement.

As in the case of the surface area measurement, it is also necessary to know the actual frame size in order to set an appropriate scale. However, in this case, we do not apply a rule but a gauge of a known length and the temperature that should be considerably different from the temperature of the examined wound. 
If we indicate the actual frame size, we can easily calculate the area of each pixel comprising a thermogram. Counting the pixels of a given temperature (or from the given temperature range) we will calculate the surface area of the wound fragment in question. The areas of skin necrosis have the temperature significantly lower than the temperature of a healthy tissue (being the point of reference) but the inflammatory condition developing in the wound increases it. An example measurement of a wound surface area is shown in the fig. 5.

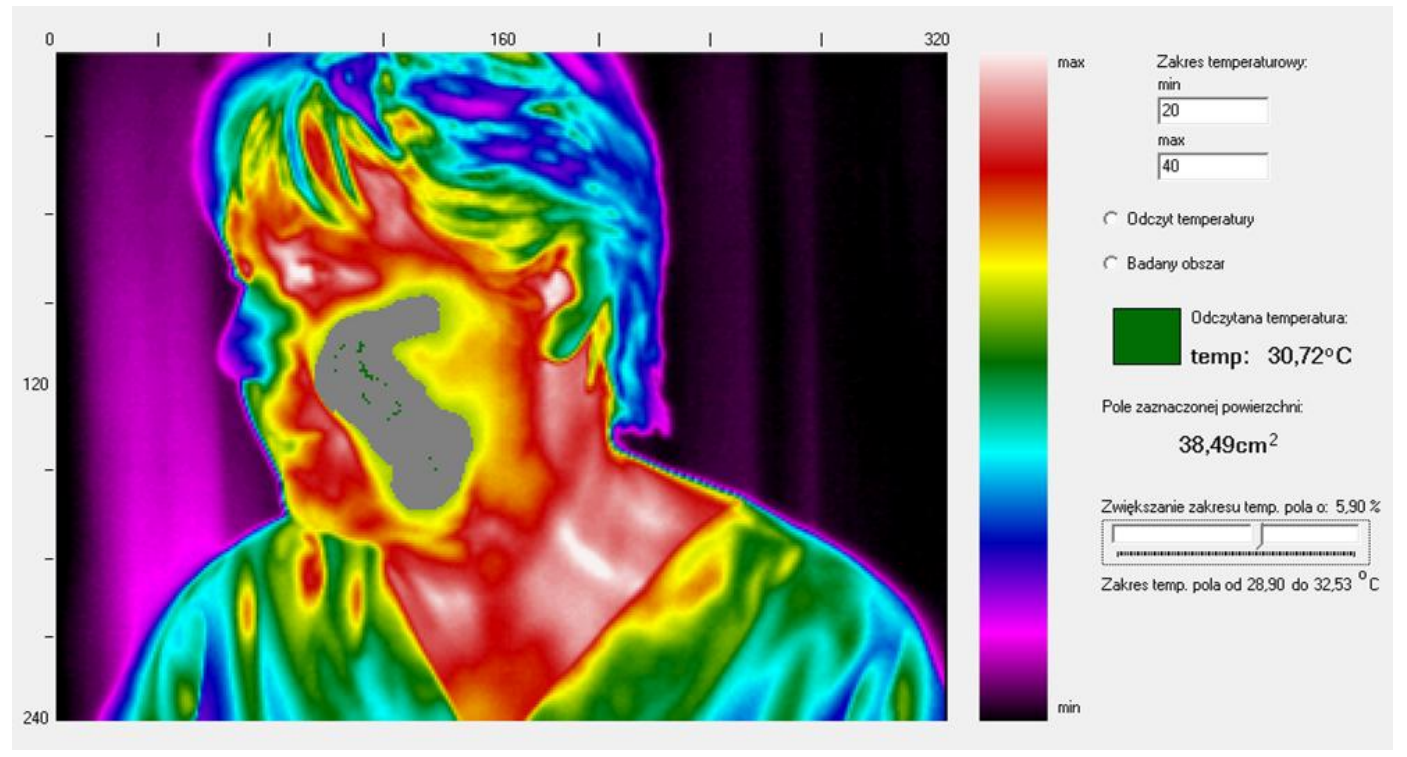

Figure 5. An example of measurement of the area on the thermogram.

\section{THERMOGRAMS RECORDING SYSTEM}

To increase the accuracy of measurement of the wound surface area on thermograms, a system that records the encircling thermograms was designed [3].

The idea of recording the encircling thermograms lies in the fact that the infrared camera records the temperature of points on the body within the strip more than ten pixels wide and the preset length $L$, while the recording will be conducted continuously at a specified frequency, when the camera moves around at a constant angular velocity. After recording the strip with a width $L$ in this way, the camera moves linearly by the same section with a necessary overlap and the system records another body strip (fig. 6).

The data collected in this way are formed in a thermal image which corresponds to a temperature distribution field projected to a plane. Both the angle of camera movement and the total length of linear displacement are adjustable.

Because used cameras do not offer a stepless depth-of-field (DOF) adjustment, it will be necessary to continuously track and change the camera position to ensure a constant distance (e.g. $250 \mathrm{~mm}$ ) from the patient's body. Tracking the distance between the camera and the patient will be provided by rangefinders that ensure a contactless distance measurement to any surface. In addition, the use of rangefinders makes it possible to avoid collisions with the patient under examination. 


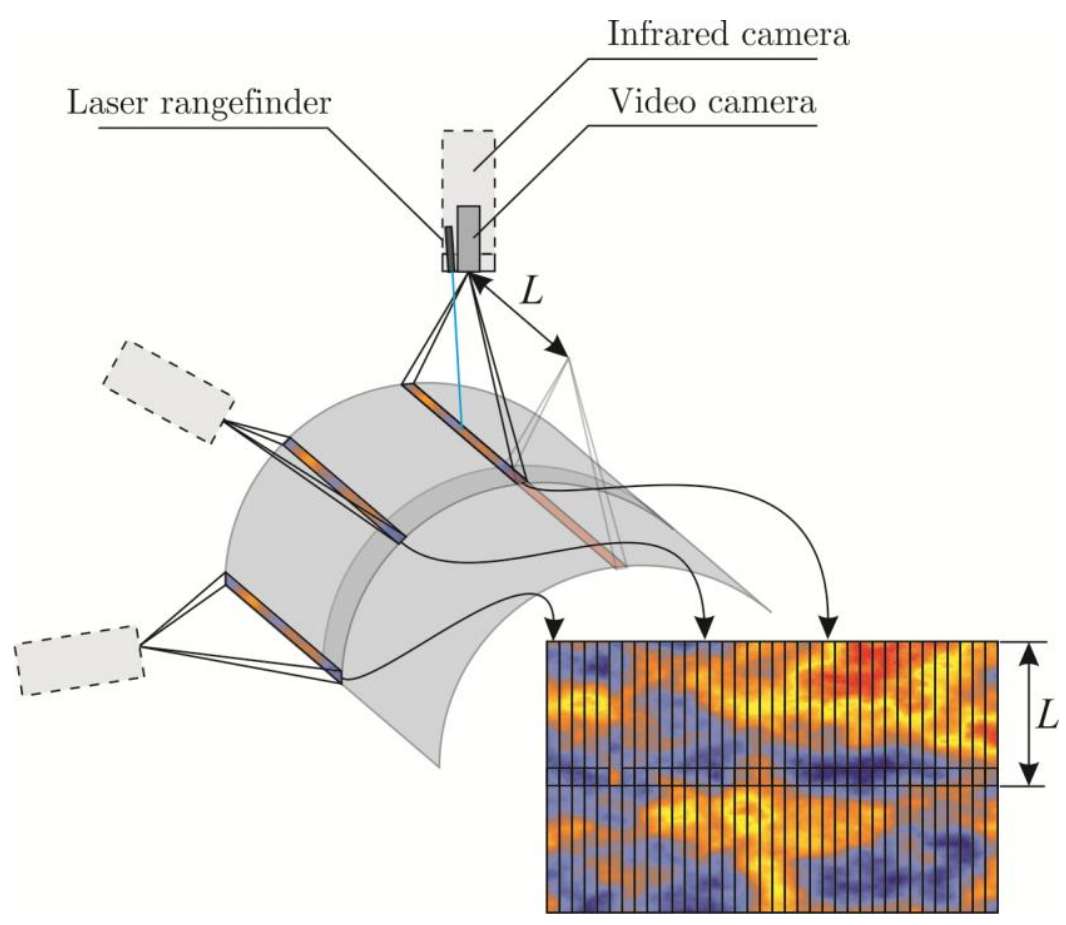

Figure 6. Visualization of the idea of recording encircling thermograms.

Within the research project, a model of a prototype device used for recording encircling thermograms was created (fig. 7). Tests performed on the device model allowed for recording clear and sharp thermal images even at high recording speeds, as it took between ten and twenty seconds to scan one strip on the trunk. Tests during recording the thermal images also included the stabilization of distance between the camera and the scanned surface and it was confirmed that deviations from the intended distance $(250 \mathrm{~mm})$ were low and the images were recorded at a depth of field resulting from the optical properties of both cameras.

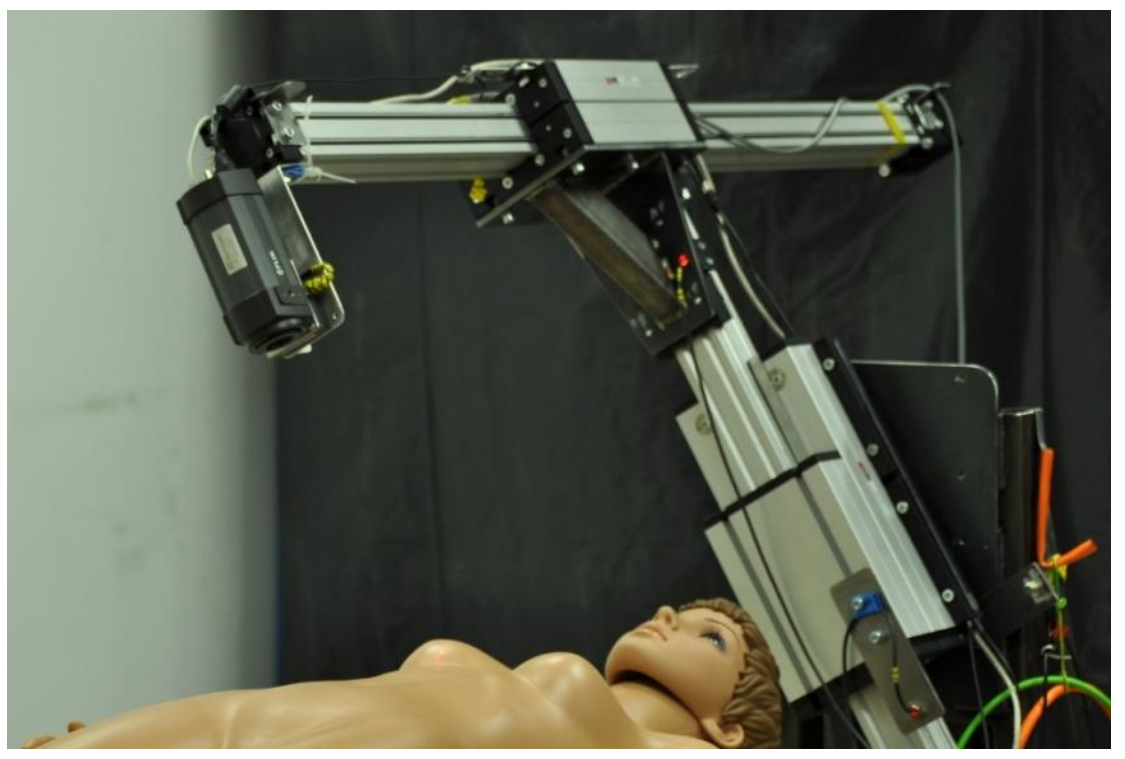

Figure 7. Model of device that records the encircling thermograms. 


\section{SUMMARY}

The assessment of wound healing process can be objectified on the basis of their surface area measurement on the images and thermograms recorded at particular stages of treatment.

Increased accuracy in measuring the wound area can be achieved using encircling thermograms.

Diagnostic system developed by consisting of a prototype device for recording encircling thermal and video images and a computer system will constitute a completely new tool intended to assist the diagnostics and the treatment process of burn and chronic wounds.

The diagnostic system developed by a team involving the author consisting of a prototype device for recording encircling thermal and video images and a computer system will constitute a completely new tool intended to assist the diagnostics and the treatment process of burn and chronic wounds. The high level of usability in clinical applications will be verified during further project stages.

\section{Acknowledgements}

The patent application covering the device presented in the article has been filed in the Polish Patent Office under the number of P.397895

The article and research were financed within the project N R13 012410 sponsored by Polish National Centre for Research and Development.

\section{REFERENCES}

[1] Dziewonski, M., "Planimetry of thermograms in diagnosis of burn wounds". Scientific Research of the Institute of Mathematics and Computer Science, Politechnika Czestochowska, Czestochowa, 33-38, 2009.

[2] Kloppenberg F., Beerthuizen G., ten Duis H., "Perfusion of burn wound assessed by laser doppler imaging in related burn depth and healing time". Burns 27, 359-363, 2001.

[3] Majchrzak E., Dziewonski M., Pietka, Nowak M., Kawecki M., Bachorz M., Kowalski P., "The design of a system for assisting burn and chronic wound diagnosis". Information Technologies in Biomedicine, 2012 (in printing).

[4] Monstrey S., Hoeksema H., Verbelen J., Pirayesh A., Blondeel P., " Assessment of burn depth and burn wound healing potential". Burns 34, 761-769, 2008.

[5] Nowakowski A. (ed.), "Advances in thermography - medical applications". Wydawnictwo Gdanskie, Gdansk, 2001 (in polish).

[6] Renkielska A., Nowakowski A., Kaczmarek M., Dobke M., Grudzinski J., Karmolinski A., Stojek W., "Static thermography revisited - An adjunct method for determining the depth of the burn injury". Burns 31, 768-775, 2005.

[7] Renkielska A., Nowakowski A., Kaczmarek M., Ruminski J., "Burn depth evaluation based on active dynamic IR thermal imaging - a preliminary study". Burns 32, 867-875, 2006.

[8] Wiecek B., Mey G.D., "Thermovision in infrared. Bases and applications". Wydawnictwo PAK, Warszawa, 2010 (in polish). 